\title{
ZONAS HOMOGÉNEAS PARA MANEJO ESPECÍFICO POR SITIO EN MAIZ USANDO UN SENSOR DE INDUCCIÓN ELECTROMAGNÉTICA EN LA SABANA DE BOGOTÁ
}

\section{HOMOGENEOUS ZONES FOR SITE-SPECIFIC MANAGEMENT IN MAIZE USING AN ELECTROMAGNETIC INDUCTION SENSOR AT THE BOGOTA SABANA}

\author{
Ricardo Alfaro Rodríguez ${ }^{1}$, Fabio Rodrigo Leiva², Manuel Iván Gómez ${ }^{3}$
}

${ }^{1}$ Agrólogo, M.Sc., c.Doctor, Catedrático. Universidad de Ciencias Aplicadas y Ambientales U.D.C.A, e-mail: ralfaror@unal. edu.co; ${ }^{2}$ Ingeniero Agrícola, M.Sc, Ph.D, profesor Titular, Facultad de Ciencias Agrarias, Departamento de Desarrollo Rural. Universidad Nacional de Colombia, Bogotá, Colombia, e-mail: frleivab@unal.edu.co; ${ }^{3}$ Ingeniero Agrónomo, M.Sc., profesor Asociado, Facultad de Ciencias Agrarias, Departamento de Agronomía, Universidad Nacional de Colombia, Bogotá, Colombia, e-mail: igomezm@unal.edu.co

Rev. U.D.C.A Act. \& Div. Cient. 18(2): 373-383, Julio-Diciembre, 2015

\section{RESUMEN}

La agricultura de precisión busca mejorar la eficiencia productiva, a partir de la variabilidad del agroecosistema. Para ello, se deben delimitar zonas homogéneas de manejo (ZM), dentro del lote de cultivo. Los sensores de inducción electromagnética (IM), que registran la conductividad eléctrica aparente (CEa), permiten identificar variaciones en propiedades del suelo y, por consiguiente, definir, en forma rápida y confiable, zonas con características similares. La investigación tuvo como objetivo delimitar ZM, con fines de manejo específico por sitio (MES), usando un sensor IM en suelos productores de maíz (Zea mays L.), de la Sabana de Bogotá. Para ello, se escogieron dos lotes en los que se sembró maíz, en los municipios de Facatativá y de Bojacá y, en estos, se midió la CEa y el contenido de agua en el suelo (Wsc). Se elaboraron mapas de CEa y Wsc y se delimitaron tres ZM, dentro de cada lote. En esas ZM, se tomaron muestras edáficas para análisis fisicoquímico de laboratorio y se determinó altura de planta, índice SPAD a los 60 y 120ddg y rendimiento del cultivo. El análisis estadístico, se realizó con SPSS V22 y SAS V 2.5. Los resultados mostraron correlaciones entre la CEa y el contenido de limo en Bojacá y el de arcilla en Facatativá y diferencias estadísticas significativas en los rendimientos de maíz entre ZM. Esta investigación destaca la utilidad del sensor IM para delimitar ZM, con fines de MES.

Palabras clave: Conductividad eléctrica aparente, agricultura de precisión, suelos agrícolas, cereales.

\section{SUMMARY}

Precision farming aims to improve production taking into account variability of the agricultural system. To do this, homogeneous management zones (HZ) are delimited within the crop field. The use of electromagnetic induction (EMI) sensors that record apparent electrical conductivity $(\mathrm{ECa})$ allows to identify variations in soil properties and in turn to define quickly and reliably zones with similar characteristics. The research had as objective ZM delimitation for site-specific management (SSM) using an EMI sensor in soils under maize crops (Zea mays L.) at the Sabana de Bogotá. For this, two agricultural fields were chosen in Facatativá and Bojacá, and in them it was measured ECa and soil water content (Wsc). Maps of ECa and Wsc were drawn and three ZM were defined within each field. In these ZM, soil samples were taken for physicochemical laboratory analysis and it was determined plant height, SPAD index at 60 and 120 DDG and crop yield. Statistical analysis was performed with SPSS V22 and SAS $\mathrm{V}$ 2.5. The results showed correlations between ECa and content of silt in Bojacá and clay content in Facatativá, and statistically significant differences in maize yields between the $Z M$. This research highlights the utility of the IM sensor to define ZM for SSM.

Key words: Apparent electrical conductivity, precision agriculture, agricultural soils, cereals.

\section{INTRODUCCIÓN}

La agricultura de precisión (AP) busca optimizar el proceso productivo, a partir del manejo de la variabilidad del agroeco- 
sistema (Dobermann et al. 2004; Bongiovanni et al. 2006; Leiva, 2008). La AP permite un mayor conocimiento y realizar trazabilidad del proceso productivo, facilita la utilización eficiente de los insumos y puede mejorar la productividad, aportando beneficios técnicos, ambientales y económicos (Roberts et al. 2010). Igualmente, la AP conduce a reducir la incertidumbre, brindando información valiosa para el mejoramiento de los sistemas productivos (Cook et al. 2003).

Para la AP, se debe identificar y cuantificar la variabilidad del agroecosistema, definir zonas homogéneas de manejo (ZM), al interior de las cuales, se usa la misma dosis de un insumo y establecer sistemas de manejo específico por sitio (MES), para las diferentes prácticas de cultivos, como son la labranza, la siembra, la fertilización y el riego (Ortega \& Flores, 1999; Leiva et al. 2013).

La conductividad eléctrica aparente (CEa), entendida como la capacidad de la masa de suelo sin disturbar para conducir la corriente eléctrica, viene siendo usada, a nivel internacional, para definir ZM, puesto que puede suministrar información acerca de importantes propiedades físicas y químicas del suelo, como son salinidad, contenido de agua del suelo (Wsc), contenido y mineralogía de arcillas, capacidad de intercambio catiónico (CIC) y tamaño y distribución de poros (McNeill, 1992; Corwin \& Lesch, 2005b). La CEa también ha mostrado ser un indicador de rendimiento en maíz, particularmente, cuando el Wsc ha sido un factor limitante para el cultivo (Sudduth et al. 2003). Así, la CEa puede ser usada para identificar ZM en lotes agrícolas y cuando se evalúa conjuntamente con datos de rendimiento del cultivo, se mejora la confiabilidad en la identificación de ZM (Anderson-Cook et al. 2002). Valga destacar que en lotes donde la CEa depende principalmente de la textura, por ejemplo, en suelos no salinos, los patrones de distribución espacial de las ZM tienden a ser estables en el tiempo, lo cual, facilita su manejo.

Los sensores que registran la CEa, de manera georreferenciada, se usan para crear mapas, con diversas zonas que presentan diferencias en la capacidad del suelo para conducir la corriente eléctrica, lo cual, se relaciona principalmente con la salinidad, el contenido de agua y nutrientes del suelo (Adamchuk, 2011). Se recomienda usar estos sensores para elaborar el plan de muestreo de los suelos, por ser un método confiable y rápido para identificar la variabilidad espacial de las propiedades físico-químicas de los suelos, asociada con la calidad del mismo y con las variaciones en el rendimiento del cultivo (Johnson et al. 2003; Corwin et al. 2003).

La CEa, se puede determinar en campo usando sensores de medición directa de resistividad eléctrica o de inducción electromagnética (IM). El equipo EM-38-MK2 trabaja en el modo IM sobre la superficie del suelo sin entrar en contacto con este y puede determinar la CEa hasta $1000 \mathrm{mS}^{-1} \mathrm{~m}^{-1}$, a profundidades en la posición vertical del sensor, en el rango de $1,5 \mathrm{~m}$ y $0,75 \mathrm{~m}$ en el perfil del suelo o bien en la posición horizontal del sensor, entre 0,75m y 0,375m (Geonics, 2012). Corwin \& Lesch (2005a) recomiendan este equipo como la primera opción para MES y estudios de calidad del suelo, destacando que la profundidad de trabajo cubre la zona completa para el desarrollo de raíces $(0-1,5 \mathrm{~m})$. La investigación tuvo como objetivo delimitar $Z M$, con fines de manejo específico por sitio (MES), usando un sensor IM en suelos productores de maíz (Zea mays L.), de la Sabana de Bogotá.

\section{MATERIALES Y MÉTODOS}

La investigación, se adelantó en dos lotes agrícolas de la Sabana de Bogotá, ubicados en los municipios de Facatativá y de Bojacá; el primero, de 7,7ha y, el segundo, de 3,5ha, en el segundo semestre de 2014. La temperatura media del área bajo estudio fluctúa entre 13,6 y $13,7^{\circ} \mathrm{C}$; la precipitación anual entre 600 y $650 \mathrm{~mm}$ y la evapotranspiración potencial, entre 630 y $640 \mathrm{~mm}$, en un paisaje de planicie fluviolacustre (IGAC, 2012).

El suelo estudiado en la localidad de Facatativá corresponde a la Consociación Talanquera (TA), clasificado taxonómicamente como Typic Haplustepts, familia: franca gruesa, mezclada, subactiva, isomésica; los suelos de esta unidad han evolucionado a partir de aluviones gruesos, son profundos, bien drenados, de reacción ligeramente ácida y fertilidad alta. El suelo en la localidad de Bojacá corresponde a la Consociación Llanitos (LL), clasificado como Pachic Haplustands, familia: medial, isomésica; la reacción del suelo es moderadamente ácida; la CIC es muy alta, a través de todos sus horizontes y la fertilidad alta (IGAC, 2012). En estos suelos, se determinó la CEa $\left(\mathrm{mS}^{-\mathrm{m}^{-1}}\right)$, usando el sensor Geonics EM-38 MK-2, mediante pases paralelos, distanciados $15 \mathrm{~m}$ entre sí, se tomó un registro por segundo, previo a la fertilización y siembra del maíz. Este sensor es de alta precisión $( \pm 0,1 \%$ del total de la escala de deflexión), liviano, con la posibilidad de operar a diversas profundidades, sin requerir contacto directo con el suelo (Sudduth et al. 2005).

Las mediciones, se hicieron usando el dipolo vertical, con un espaciamiento entre bobinas de $1 \mathrm{~m}$, con lo cual, se tiene un alcance de $1,5 \mathrm{~m}$ de profundidad en el suelo. El sensor, se ubicó sobre una carpa de lona diseñada para el arrastre sobre la superficie del suelo, a través de los lotes.

Simultáneamente, con las mediciones de CEa, se midió Wsc, con el medidor TDR 300(Spectrum Technologies, inc), usando una grilla regular de $15 \mathrm{~m}$ por $50 \mathrm{~m}$. Los datos de CEa reportados por el sensor fueron filtrados, para eliminar valores atípicos; al respecto es importante tener en cuenta que el aparato cuenta con un computador de campo, que 
geo-posiciona cada registro. A los datos obtenidos de CEa y de Wsc, se les aplicó estadística descriptiva y, luego, se generaron mapas de estas propiedades, mediante geoestadística, incluyendo análisis estructural y posterior interpolación, mediante kriging ordinario. Éste último, cuantifica la estructura espacial de los datos usando los semivariogramas y los predice estadísticamente, asumiendo que los datos más cercanos a un punto conocido tienen mayor peso en la interpolación, que aquellos más distantes (Bullock et al. 2007). El valor interpolado con este método carece de sesgo, ya que la interpolación de las variables en un punto medido devuelve el valor de la variable en ese punto, con un error de estimación casi nulo (Vieira, 2000).

Para seleccionar el modelo teórico de semivariograma de mejor ajuste, se usó validación cruzada, considerando los siguientes criterios para los errores de predicción: error medio estandarizado cercano a cero, error cuadrático medio pequeño, error estándar promedio similar al error cuadrático medio y error cuadrático medio estandarizado cercano a 1 . Con el semivariograma teórico seleccionado, se obtuvo el correspondiente mapa, usando kriging ordinario y se evaluó el mapa de errores, para garantizar que estos fueran inferiores al $10 \%$. Posteriormente, se usó cokriging, para evaluar la posible mejora del mapa de CEa a partir del encontrado para
Wsc, realizando validación cruzada, con los criterios citados de evaluación del error de predicción.

Con el fin de garantizar confiablidad, de manera similar a lo reportado por Simón et al (2013), en cada uno de los lotes se definieron tres ZM, usando quantiles, como método de clasificación. Las zonas identificadas, se denominaron "baja(b)", "media(m)" y "alta(a)", a partir de los valores encontrados de CEa, para Facatativá, los limites fueron 12,834 - 16,038(b), 16,038 - 17,405(m) y 17,405 - 24,123(a) y para Bojacá, se tuvo 6,78 - 12,092(b), 12,092 - 14,28(m) y 14,28 $-23,225(a)$.

Posteriormente, en cada ZM, se georreferenciaron puntos de muestreo, los cuales, fueron centrados dentro de las distintas zonas de CEa, para evitar espacios de transición; en éstos, se midió la CEa con el sensor IM, se tomaron muestras de suelo para análisis físico-químico de laboratorio y se efectuaron mediciones de variables de desarrollo y rendimiento del cultivo de maíz (Cuadro 1). En Facatativá, las muestras de suelo, a una profundidad entre 0 y $20 \mathrm{~cm}$, se realizaron con tres repeticiones por zona y en la ZM con mayor extensión; se analizaron cuatro muestras. En Bojacá, se tomaron tres repeticiones por ZM. Cada muestra de suelo estuvo compuesta por diez submuestras, de acuerdo a la metodología propuesta por CORPOICA (Cuesta \& Villaneda, 2005).

Cuadro 1. Propiedades físico-químicas de suelos y variables de desarrollo y rendimiento del cultivo de maíz, evaluadas en Bojacá y Facatativá.

\begin{tabular}{|c|c|}
\hline Variable & Método \\
\hline \multicolumn{2}{|l|}{ 1.1. Físicas - Suelo } \\
\hline Contenido de agua del suelo (Wsc) & Uso de medidor TDR 300 \\
\hline \multicolumn{2}{|l|}{ 1.2. Químicas - Suelo } \\
\hline Conductividad eléctrica aparente (CEa) & $\begin{array}{l}\text { Sensor Geonics EM-38MK-2. Primera medición usando transectos; } \\
\text { segunda medición, en zonas de muestreo }\end{array}$ \\
\hline Capacidad de intercambio catiónica & Extracción con acetato de amonio $1 \mathrm{M} \mathrm{pH} 7$ \\
\hline $\mathrm{pH}$ & Suspensión suelo: agua (relación peso: volumen 1:1) \\
\hline Carbono orgánico oxidable & Walkley-Black \\
\hline P Fósforo disponible & Bray II \\
\hline S Azufre & Extracción con fosfato monocálcico \\
\hline Índice SPAD de contenido de clorofila en campo & $\begin{array}{l}\text { Clorofilómetro Minolta SPAD 502, (en campo). Estado de desarrollo: } 60 \\
\text { y 120ddg* }\end{array}$ \\
\hline Altura de planta & Longitud total en cm. Estado de desarrollo: 60 y 120ddg \\
\hline Rendimiento en grano por planta & $\begin{array}{l}\text { Conteo, recolección y pesaje. Mazorca con y sin capacho en } 3 \mathrm{~m}^{2} \text {, } \\
\text { a cosecha en estado de choclo }\end{array}$ \\
\hline
\end{tabular}

\footnotetext{
* Días después de germinación.
} 
Las mediciones y los métodos de determinación de las variables de desarrollo y de rendimiento del cultivo, se describen en la cuadro 1. Las mediciones con el clorofilómetro Minolta ${ }^{\circledR}$ SPAD 502, se tomaron en una hoja por planta, en diez plantas por sitio, aproximadamente, a la mitad de la distancia entre el tallo y el ápice de la hoja y a la mitad de la distancia, entre el borde de la hoja y el nervio medio, en la última hoja expandida y en la hoja de la espiga en floración.

En cada lote, se aplicó estadística descriptiva: medidas de tendencia central, de dispersión y de distribución para las propiedades del suelo y las variables de desarrollo y rendimiento del cultivo. A partir del coeficiente de variación (CV), se evaluó la variabilidad de las propiedades del suelo, adaptando la escala propuesta por Larreal (2005): muy baja variabilidad $<15 \%$, baja variabilidad de $\geq 15$ a $<30 \%$, moderada variabilidad de $\geq 30$ a $<45 \%$, variabilidad alta de $\geq 45$ a $60 \%$ y muy alta variabilidad $>60 \%$. Luego, se evaluaron correlaciones Pearson entre estas y la CEa, medida en cada uno de los puntos de muestreo, utilizando el programa SPSS V22 (IBM); dichas correlaciones determinan si existe una relación lineal estadísticamente significativa entre dos variables, sin que esta sea debida al azar (Corwin \& Lesch, 2005a).

Para evaluar diferencias en los promedios de las variables de desarrollo y de rendimiento del cultivo, en cada localidad en las diferentes ZM, se usó el procedimiento ANOVA de PROC MIXED de SAS V 2.5, con las ZM, como efectos fijos y los puntos de muestreo dentro de cada una de estas, como una repetición (Littell et al. 1996). Para el efecto, se consideró cada ZM como un tratamiento, en un diseño de bloques completos al azar, utilizando Tukey para las pruebas de diferencia mínima significativa (DMS).

\section{RESULTADOS Y DISCUSIÓN}

\section{Análisis estructural de la CEa y el Wsc.}

Se consideraron los modelos teóricos de los semivariogramas: circular, esférico, tetraesférico, pentaesférico, exponencial, gaussiano, cuadrático rotacional, efecto hoyo, K-Bessel, J-Bessel y stable y los criterios de selección. Igualmente, se evalúo anisotropía, sin que mejoraran los errores de predicción, por lo tanto, no se consideró esta característica. El tipo y parámetros de los semivariogramas seleccionados, se ilustran en la tabla 1. Los rangos para CEa y Wsc fueron considerablemente superiores en Bojacá, indicando mayor autocorrelación espacial para esas variables, en dicha localidad. Los mapas generados, a partir de los modelos descritos, se presentan en la figura 1. Estos resultados ilustran el alto potencial del sensor de inducción electromagnética utilizado, en la delimitación de ZM, con fines de MES, en razón a su precisión, versatilidad y confiabilidad.

\section{Propiedades fisicoquímicas del suelo y de las variables de desarrollo y rendimiento del cultivo.}

Bojacá. La CEa inicial y Wsc presentaron baja variabilidad y una distribución normal con valores similares de media y mediana y coeficientes de curtosis y de asimetría inferiores a 1 (Tabla 2). La CEa, medida en cada uno de los puntos de muestreo, presentó una variabilidad moderada en razón a los rangos entre $Z M$ ya mencionados e igualmente una distribución normal.

La textura en este lote es franca $(F)$, con muy baja variabilidad para Ar, L y A. La Ar presentó una distribución normal, según la prueba de Shapiro-Wilk (Tabla 2). En razón a que L y A no presentaron normalidad, se realizaron las transformaciones pertinentes para cada una de estas, encontrándose respuesta únicamente para $\mathrm{L}$ con la expresión: $\mathrm{X}=\mathrm{Log}$ (C$\mathrm{L})$, donde $\mathrm{C}$ es una constante y $\mathrm{L} \%$ de limo; la significancia de la prueba Shapiro-Wilk para esta variable transformada fue de 0,5. La CIC, igualmente presentó muy baja variabilidad y una distribución normal, con valores altos, debido a los contenidos elevados de materia orgánica y a la naturaleza andica de los suelos, estos concuerdan con los reportados por IGAC (2012).

Con relación a las variables de desarrollo y de rendimiento del cultivo, el peso del maíz, la altura de planta a los 60 y $120 d d g$ y el índice SPAD a los 60 y 120ddg presentaron distribuciones normales, con variabilidad en el rango de muy baja a baja. El peso de la mazorca mostró baja variabilidad, similar a lo encontrado con CEa inicial y una distribución ligeramente leptocúrtica.

Facatativá. La CEa inicial presentó variabilidad muy baja, mientras que la CEa y Wsc, medida en cada uno de los puntos de muestreo, expusieron baja variabilidad; en general, a mayor cantidad de agua en el suelo mayor conductividad eléctrica, en razón al efecto electrolítico del agua, ya que disuelve las sales presentes (Corwin \& Lesch, 2005a). Estas variables presentaron una distribución normal.

Las propiedades físico-químicas del suelo L y CIC con muy baja variabilidad y $\mathrm{Ar}$ con variabilidad baja presentaron una distribución normal; de otro lado, la A mostró una distribución que no corresponde a la normal, ni siquiera mediante transformación de los datos originales. La textura para este suelo resultó franca $(F)$, de manera similar a lo encontrado en el lote de Bojacá.

En cuanto a las variables de rendimiento y desarrollo del cultivo todas presentaron distribuciones normales y variabilidad de baja a muy baja, que fluctúan entre $29,7 \%$, para el peso del maíz y $11,03 \%$, para el índice SPAD a los 120ddg. 


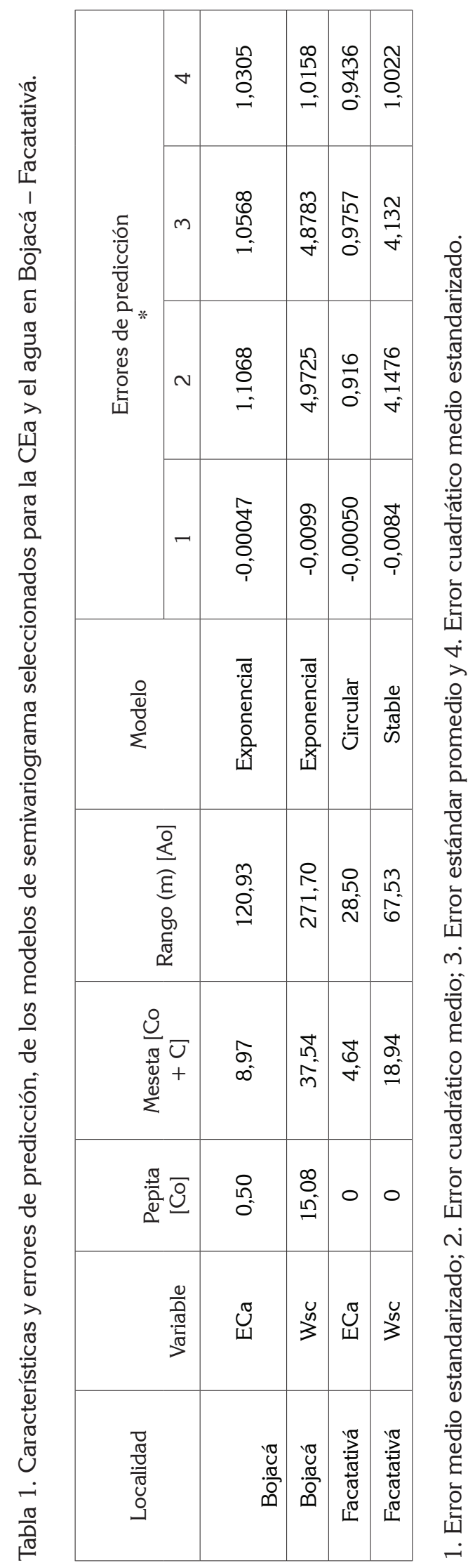



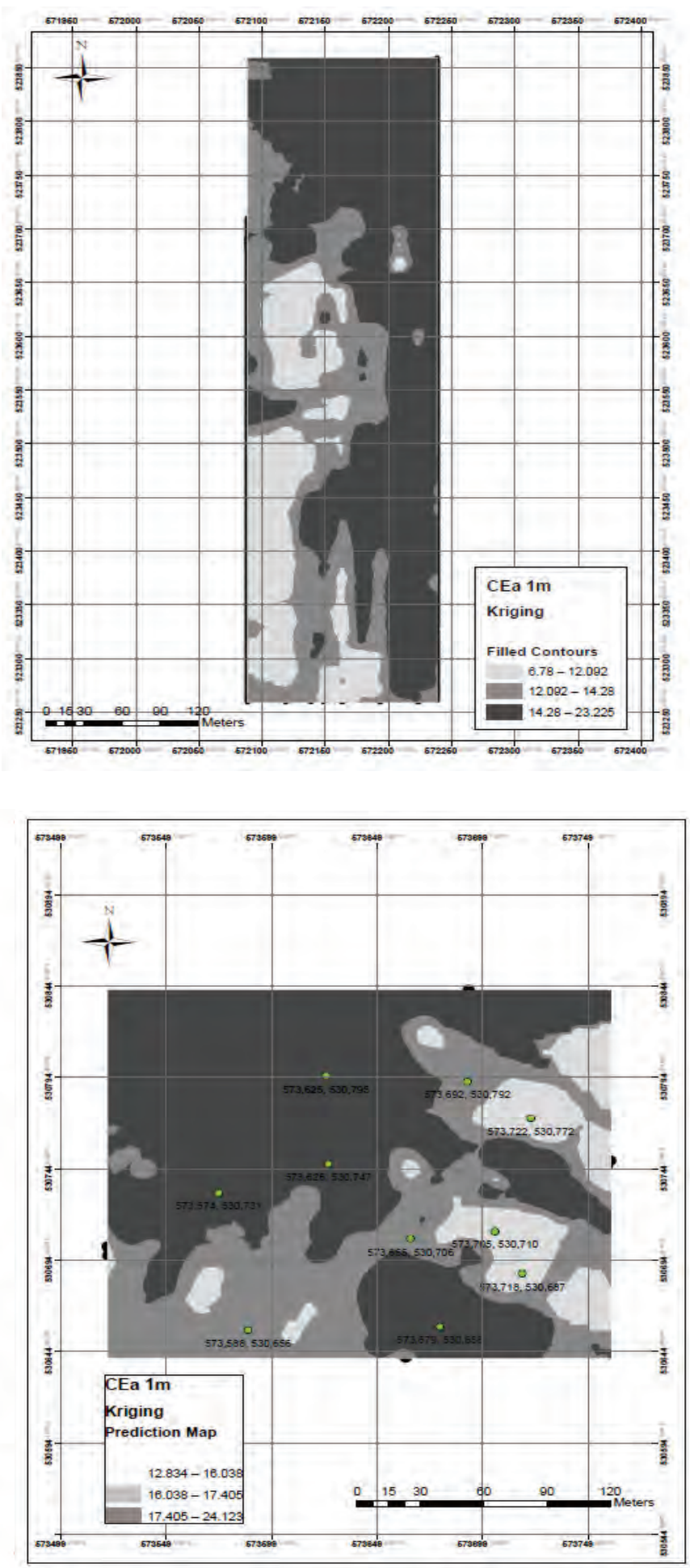

Figura 1. Mapas de CEa del suelo en los lotes experimentales. Bojacá (arriba) - Facatativá (abajo), con los puntos de muestreo dentro de las ZM, 2014. 


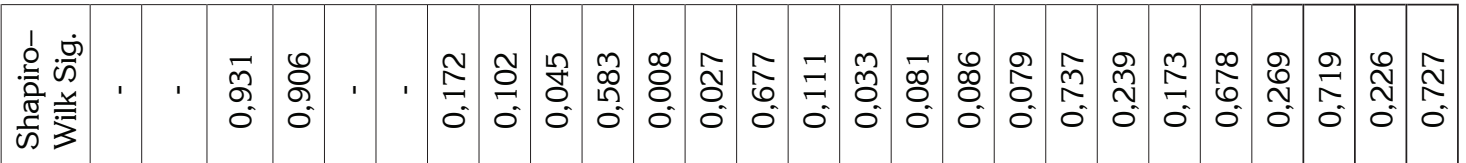

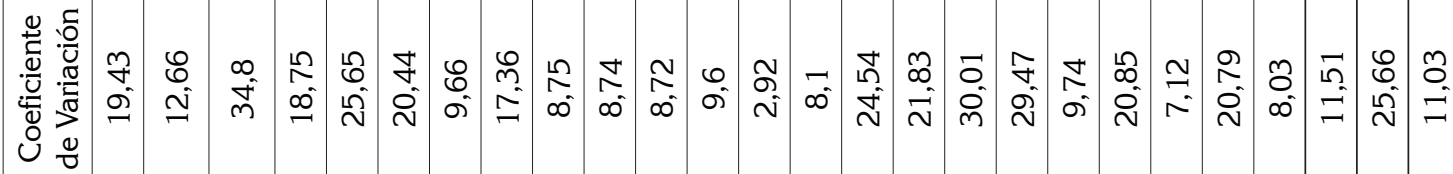

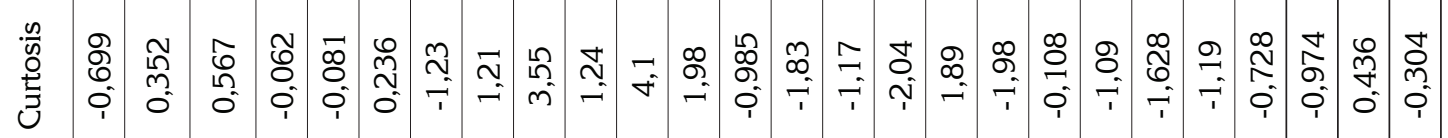

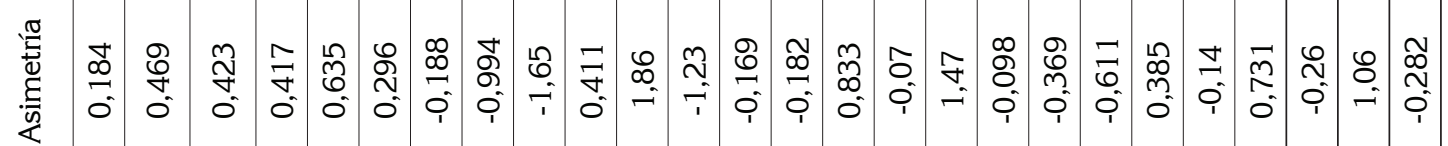

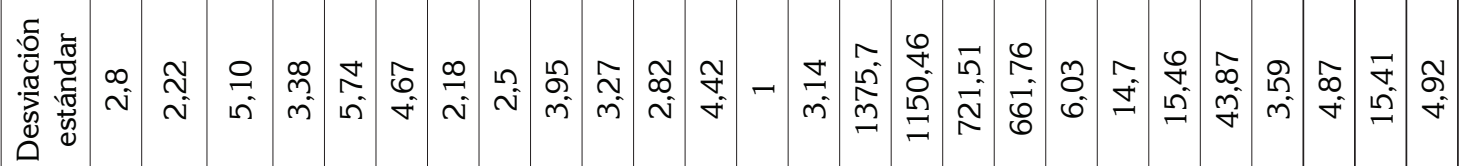

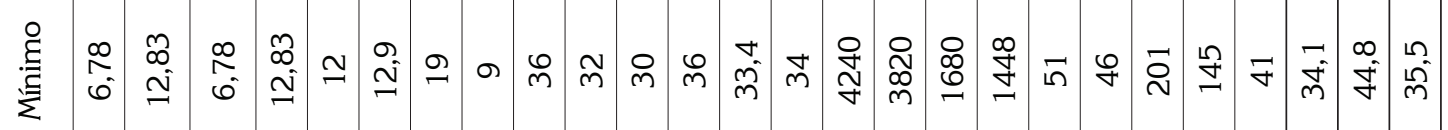

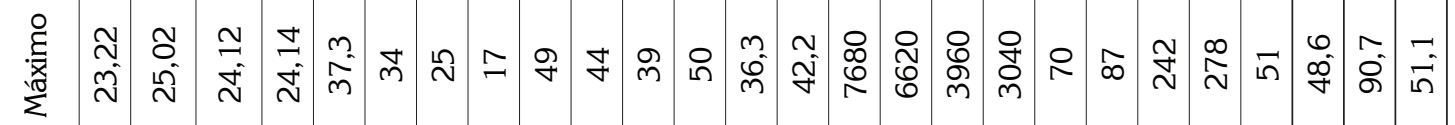

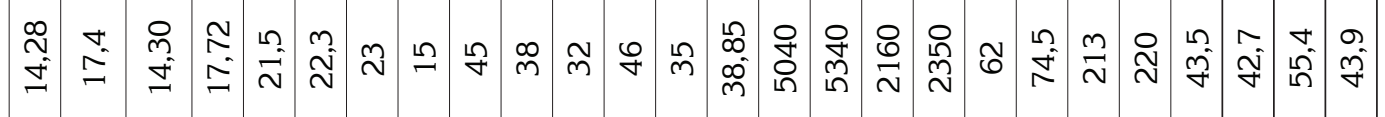

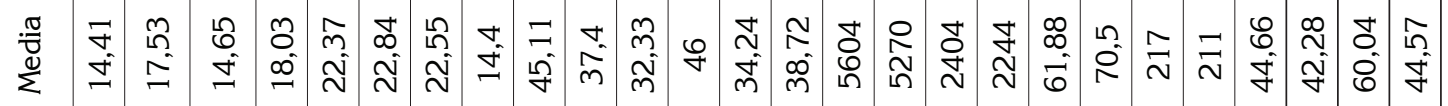

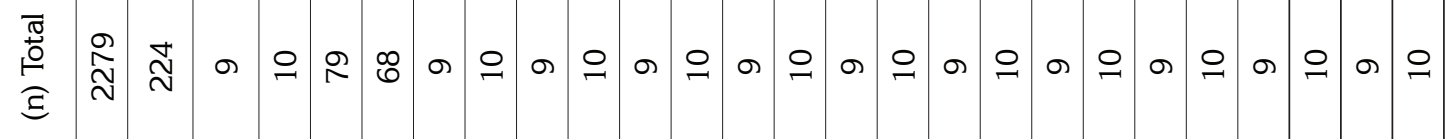
$\frac{\substack{0 \\ \frac{\pi}{0}}}{\frac{\pi}{0}}$ の

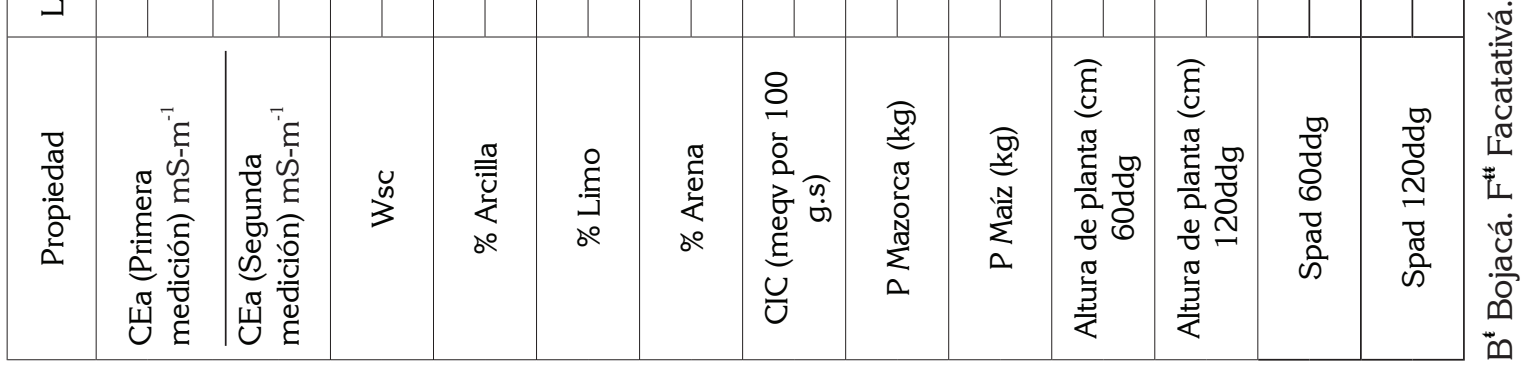




\section{Relaciones entre variables.}

Bojacá. Este análisis mostró una correlación Pearson altamente significativa, con un coeficiente de asociación de 0,846 , entre la CEa, medida en cada uno de los puntos de muestreo y el contenido de $L$ del suelo y de $-0,813$, entre esta y la Ar. Esta correlación encontrada entre la CEa y el L es consistente con resultados de otros investigadores (Sudduth et al. 2003; Corwin \& Lesch, 2005a) e indica el efecto de la textura en la CEa. Si bien, la Ar tiene mayor conductividad eléctrica que el L (Grisso et al. 2007), probablemente, el predominio de este último, en este caso, con un contenido que duplica al de $\operatorname{Ar}(45,1 \%$ frente a $22,5 \%)$ y, adicionalmente, la muy baja variabilidad del contenido de Ar, a través del lote, conllevó a un mayor efecto del L en la CEa. Las correlaciones de la CEa con las otras propiedades físico-químicas evaluadas no fueron significativas, indicando que la variabilidad espacial de esta, fue función principalmente de la textura del suelo (Corwin \& Lesch, 2005b).

Con respecto a las variables de desarrollo y de rendimiento, se encontró correlación significativa de 0,720 entre el peso de la mazorca y la CEa. Cuando los rendimientos correlacionan con la CEa, las medidas espaciales de esta, se pueden usar en el MES del cultivo, para lo cual, es necesario establecer las propiedades del suelo que tienen mayor influencia en la CEa, dentro del campo y, así mismo, definir si estas mismas son las que más influyen sobre los rendimientos (Corwin et al. 2003). Lo hallado indica que las ZM identificadas, efectivamente, fueron útiles en términos de rendimiento del cultivo.
No hubo correlación de la CEa con las demás variables de desarrollo y de rendimiento. En el caso del índice SPAD, la medición resulta de gran importancia en la evaluación indirecta del contenido de clorofila de la hoja (Blackmer \& Schepers, 1995); sin embargo, no presentó correlación con el rendimiento, probablemente, porque este índice no considera el área foliar; además, los investigadores citados confirman la baja correlación existente entre los valores SPAD y el rendimiento del cultivo de maíz, destacando que su uso, como herramienta de diagnóstico, tiene un limitado potencial, que podría mejorar en estadios fenológicos posteriores.

Los diagramas de caja que se presentan en la figura 2, ilustran lo expuesto frente al contenido de L, que explica, en gran medida, los cambios de CEa, a través del lote y la respuesta del cultivo en cada una de las tres ZM delimitadas, con base en la CEa. La prueba de Anova (Tabla 3) realizada al peso de la mazorca, arrojó diferencias altamente significativas entre tratamientos, lo cual, confirma la respuesta diferencial del cultivo en cada una de las ZM, con promedios de 4426,66g (a), en zona "baja"; 5026,66g (b), en zona "media" y 7360,80g (c), en la zona "alta" de CEa.

Facatativá. En este lote, se encontró correlación Pearson significativa con un coeficiente de asociación de 0,758 , entre la CEa, medida en cada uno de los puntos de muestreo y el contenido de Ar del suelo y entre este y la altura de plantas, a los $120 \mathrm{ddg}$ de 0,654 . La correlación positiva encontrada con la Ar es coherente con estudios previos (Sudduth et al. 2003) y, posiblemente, se debe a la mayor variabilidad de esta propiedad en relación con L, a través del lote y al menor con-

Tabla 3. Anova para rendimiento de la mazorca y altura de plantas de maíz 120ddg en Bojacá y Facatativá.

\begin{tabular}{|c|c|c|c|c|c|c|}
\hline \multicolumn{2}{|l|}{ FV } & GL & SC & $C M$ & $\mathrm{~F}$ & $\mathrm{P}>\mathrm{F}$ \\
\hline \multirow[b]{2}{*}{ TRATAMIENTO } & R-M* & 2 & $14^{\prime} 408.896 .00$ & 7'204.448.00 & 66,92 & 0,002 \\
\hline & A-P** & 2 & 13.446 .21 & 6.723 .10 & 13,83 & 0,018 \\
\hline \multirow[b]{2}{*}{ BLOQUE } & R-M & 2 & 301.120 .00 & 150.560 .00 & 1,39 & 0,347 \\
\hline & A-P & 2 & 797.56 & 394.78 & 0,82 & 0,505 \\
\hline \multirow[b]{2}{*}{ ERROR } & R-M & 4 & 430.592 .00 & 107.648 .00 & & \\
\hline & A-P & 4 & 1.944 .43 & 486.10 & & \\
\hline \multirow[b]{2}{*}{ TOTAL } & R-M & 8 & $15^{\prime} 140.608 .00$ & & & \\
\hline & A-P & 8 & 16.188.21 & & & \\
\hline
\end{tabular}

C.V RM $=5,85 \% *$ Peso de la mazorca

C.V AP $=10,28 \% * *$ Altura de planta 

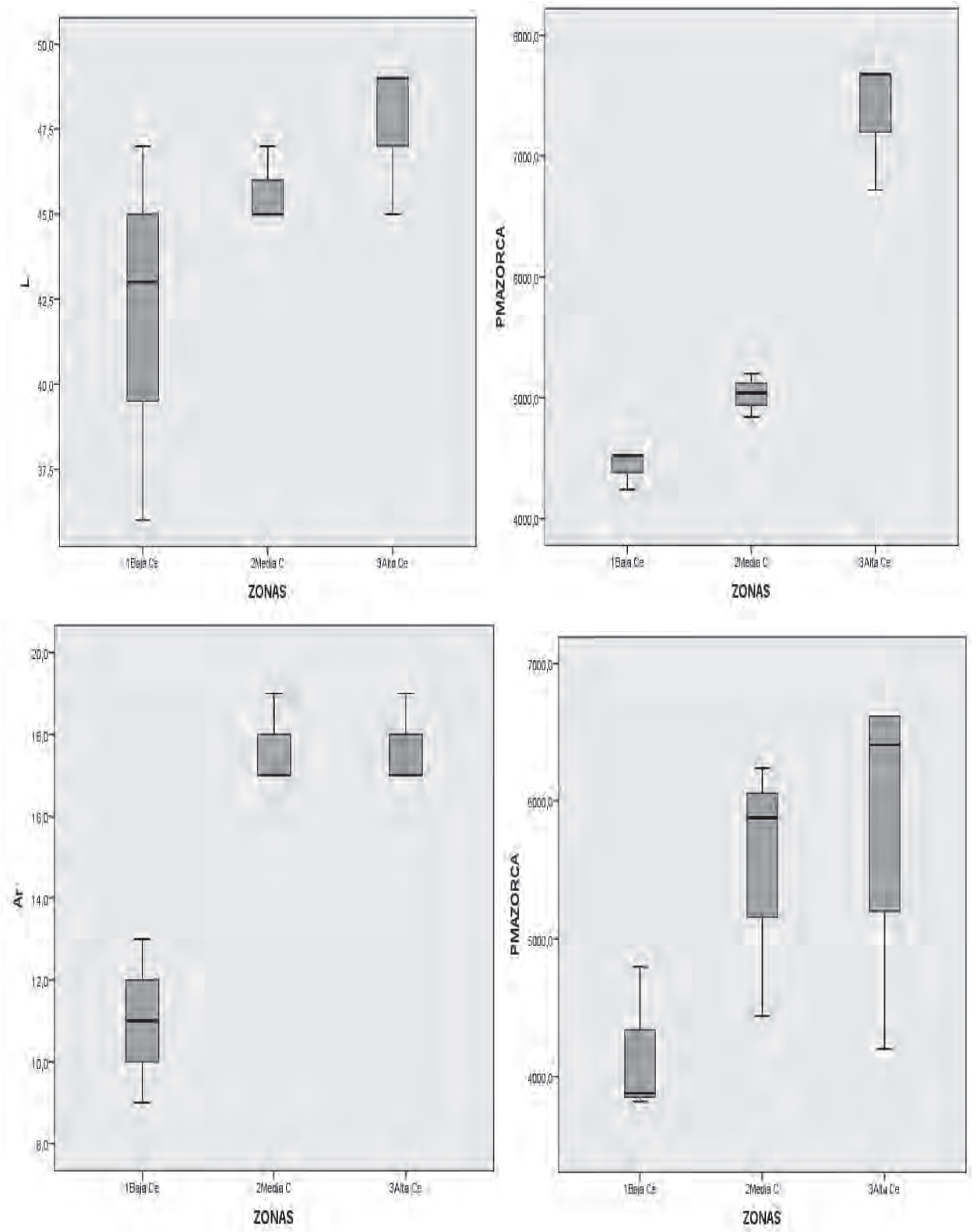

Figura 2. Diagramas de caja por zonas para la comparación de medias de: contenido de limo y peso de la mazorca en Bojacá (arriba) y contenido de arcilla y peso de la mazorca en Facatativá (abajo), 2014.

tenido de L, en comparación con el de Bojacá. La CEa no presentó correlación significativa con las variables de rendimiento y de desarrollo; vale la pena destacar el coeficiente de 0,606 , entre la CEa y el peso de la mazorca y de 0,485, entre este último y el contenido de Ar. Las variables agronómicas que mostraron correlaciones altamente significativas entre sí fueron el peso de la mazorca y la altura de las plantas, a los 60 y $120 \mathrm{ddg}$, con valores de 0,798 y 0,852, respectivamen- te. De otro lado, la medición de SPAD presentó correlación significativa de 0,643 con la altura de planta, a los 60ddg.

Los diagramas de caja que se presentan en la figura 2, ilustran un claro contraste entre la zona "baja" y las otras dos zonas, en términos de Ar, por mayor contenido, lo cual, se vio reflejado en la respuesta del cultivo. 
Las correlaciones entre la CEa y los contenidos de Ar y L en los lotes estudiados indican que la CEa, en estos suelos, depende, principalmente, de las fracciones finas de la textura, que brinda cierta estabilidad espacial y temporal a las ZM delimitadas y facilita el MES.

La prueba de Anova (Tabla 3), realizada a la altura de planta a los $120 \mathrm{ddg}$, arrojó diferencias altamente significativas entre tratamientos, lo que confirma la respuesta diferencial del cultivo en cada una de las ZM delimitadas, con promedios de 1,603m (a), en zona "baja"; 2,357m (b), en zona "media" y $2,477 \mathrm{~m}$ (c), en la zona "alta" de CEa. Por la alta correlación existente entre la altura de las plantas a los $120 \mathrm{ddg}$ y el peso de la mazorca, se puede asegurar que esta variable agronómica es un buen indicador del rendimiento del cultivo. La importancia de la altura de la planta se debe a que es un parámetro que determina el grado de desarrollo del área foliar y el tamaño final de la planta, en algunos casos, el mayor tamaño de una planta es más importante que la duración del período de llenado de grano, en la determinación del rendimiento (Fassio et al. 1998).

En los dos lotes, las ZM delimitadas con base en la CEa presentaron diferencias significativas: uno, en la respuesta de altura de planta y, el otro, en el rendimiento del cultivo de maíz. Esto permite concluir que el cultivo mostró respuesta diferencial concordante con los rangos de CEa, que sirvieron para delimitar las ZM.

Los suelos estudiados presentan alta fertilidad; sin embargo, es común encontrar desbalances en los contenidos de nutrientes, que obligan a realizar aplicaciones de fertilizantes o enmiendas con miras a restablecer el equilibrio, por ejemplo, balance en la saturación de $\mathrm{K}$, manejo en las relaciones $\mathrm{Ca} /$ $\mathrm{Mg}$. Es recomendable que su manejo técnico se enmarque en el contexto de la AP, como lo muestran otros estudios, que han reportado bondades técnicas y económicas de definir ZM para fertilización específica por sitio, en cultivos de maíz en la Sabana de Bogotá (Rodríguez et al. 2008).

Los resultados hallados permiten asegurar que el uso del sensor de IM posee gran potencial en la identificación de ZM, para MES en el cultivo de maíz.

Conflictos de intereses: El manuscrito fue preparado y revisado con la participación de todos los autores, quienes declaramos que no existe conflicto de intereses que ponga en riesgo la validez de los resultados presentados. Financiación: Este estudio fue financiado por la Universidad Nacional de Colombia, sede Bogotá, dirección de Investigaciones y hace parte del Proyecto: "Metodología basada en el uso de un sensor de conductividad eléctrica aparente (CEa) para la zonificación con fines de fertilización específica por sitio en suelos productores de maíz (Zea mays L.) de la Sabana de Bogotá".

\section{BIBLIOGRAFÍA}

1. ADAMCHUK, V. 2011. On-the-go soil sensors - are we there yet? Ed. McGill University. Ste-Anne-de-Bellevue. Quebec (Canada). p.63.

2. ANDERSON-COOK, C.; ALLEY, J.; ROYGARD, R.; KHOSLA, R.; NOBLE, R.; DOOLITTLE, J. 2002. Differentiating soil types using electromagnetic conductivity and crop yield maps. Soil Sci. Soc. Am. J. 66:1562-1570.

3. BLACKMER, T.; SCHEPERS, J. 1995. Use of a chlorophyll meter to monitor nitrogen status and schedule fertigation for corn. J. Prod. Agr. (USA). 8:56-60.

4. BONGIOVANNI, R.; MANTOVANI, E.; BEST, S.; ROEL, Á. 2006. Agricultura de precisión: Integrando conocimientos para una agricultura moderna y sustentable. Ed. Instituto Interamericano de Cooperación para la Agricultura (IICA). (Uruguay). 244p.

5. BULLOCK, D.; KITCHEN, N. 2007. Multidisciplinary teams: a necessity for research in precision agriculture systems. Crop Sci. 47:1765-1769.

6. COOK, S.; OBRIEN, R.; CORNER, R.; OBERTHUR, T. 2003. Is precision agriculture irrelevant to developing countries? En: Stafford, J.; Werner, A. (eds). Precision agriculture. Wageningen Academic Publishers. Netherlands (Holanda). p.115-119.

7. CORWIN, D.; LESCH, S. 2005a. Characterizing soil spatial variability with apparent soil electrical conductivity: Part II. Case study. Computers Electronics Agric.46:135-152.

8. CORWIN, D.; LESCH, S. 2005b. Characterizing soil spatial variability with apparent soil electrical conductivity I. Survey protocols Computers Electronics Agric. 46:103-133.

9. CORWIN, D.; LESCH, S.; SHOUSE, P.; SOPPE, R.; AYARS, J. 2003. Identifying soil properties that influence cotton yield using soil sampling directed by apparent soil electrical conductivity. Agron. J. 95(2):352-364.

10. CUESTA, P.; VILLANEDA, E. 2005. El análisis de suelos: toma de muestras y recomendaciones de fertilización para producción ganadera. En: Manual Técnico Producción y Utilización de Recursos Forrajeros en Sistemas de Producción Bovina en las regiones Cari- 
be y Valles Interandinos. CORPOICA. (Bogotá D.C). 96p.

11. DOBERMANN, A.; BLACKMORE, S.; COOK, S.; ADAMCHUK, V. 2004. Challenges and future directions. En: Krishna, K, (ed). Precision farming: Soil fertility and productivity aspects. Ed. Apple Academis Press Inc. (USA). p.71-90.

12. FASSIO, A.; CARRIQUIRI, A.; TOJO, C.; ROMERO, R. 1998. Maíz aspectos sobre fenología. INIA. Montevideo (Uruguay). 59p.

13. GEONICS EM38K2. 2012. Ground conductivity meter operating manual. Geonics Limited. Leaders in electromagnetics. Mississaagua (Ontario). 57p.

14. GRISSO, R.; ALLEY, M.; HOLSHOUSER, D.; THOMASON, W. 2007. Precision farming tools: Soil electrical conductivity. Virginia. Cooperative Extension. p.442-508.

15. INSTITUTO GEOGRÁFCO AGUSTÍN CODAZZI. -IGAC2012. Levantamiento Detallado de Suelos en las Áreas Planas de 14 municipios de la Sabana de Bogotá. IGAC. Bogotá D.C. 492p.

16. JOHNSON, C.; DORAN, J.; DUKE, H.; WEINHOLD, B.; ESKRIDGE, K.; SHANAHAN, J. 2003. Field scale electrical conductivity mapping for delineating soil condition. Soil Sci. Soc. Am. J. 65:1829-1837.

17. LARREAL, R. 2005. Definición y establecimiento de la serie San Francisco en la altiplanicie de Maracaibo, sector semiárido. Facultad de Agronomía. Luz. Maracaibo. (Venezuela). 106p.

18. LEIVA, F.; CRIOLLO, V.; GUERRERO, L. 2013. Aproximación al riego por sitio específico: Estudio de caso en la Sabana de Bogotá. Suelos Ecuatoriales. (Colombia). 43(2):82-86.

19. LEIVA, F. 2008. Agricultura de precisión en cultivos transitorios. Universidad Nacional de Colombia. Bogotá. D.C. $107 p$.
20. LITTELL, R.; MILLIKEN, G.; STROUP, W.; WOLFINGER, R. 1996. SAS System for mixed Models, Cary, NC: SAS Institute Inc. 596p.

21. McNEILL, J. 1992. Rapid, accurate mapping of soil salinity by electromagnetic ground conductivity meters. In: Topp, G.C.; Reynolds, W.D.; Green, R.E. (eds). Advances in measurement of soil physical properties: Bringing theory into practice. SSSA Spec. Madison, (WI). p.209-229.

22. ORTEGA, R.; FLORES, L. 1999. Agricultura de precisión: introducción al manejo sitio-específico. Ministerio de Agricultura, Instituto de investigaciones agropecuarias. CRI Quilamapu. (Chile). p.13-46.

23. ROBERTS, D.; KITCHEN, N.; SUDDUTH, K.; SHARF, P. 2010. Will variable-rate nitrogen fertilization using corn canopy reflectance sensing deliver environmental benefits. Agronomy. J. 102:85-95.

24. RODRÍGUEZ, J.; GONZÁLEZ, A.; LEIVA, F.; GUERRERO, L. 2008. Fertilización por sitio específico en un cultivo de maíz (Zea mays L.) en la Sabana de Bogotá. Agr. Col. 26(2):308-321.

25. SIMÓN, M.; PERALTA, N.; COSTA, J. 2013. Relación entre la conductividad eléctrica aparente con propiedades del suelo y nutrientes. Ciencia Suelo (Argentina). 31(1):45-55.

26. SUDDUTH, K.; KITCHEN, N.; WIEBOLD, W. 2005. Relating apparent electrical conductivity to soil properties across the north-central USA. Computers and Electronics in Agriculture. 46(1-3):263-283.

27. SUDDUTH, K.; KITCHEN, N.; BOLLERO, G.; BULLOCK, D.; WIEBOLD, W. 2003. Comparison of electromagnetic induction and direct sensing of soil electrical conductivity. Agron. J. 95:472-482.

28. VIEIRA, S. 2000. Uso de geoestadística en estudios de variabilidad espacial de propiedades del suelo. Tópicos em Ciência do Solo. Viçosa. Sociedade Brasileira de Ciência do Solo. Ed. Novais. 87p.

Recibido: Mayo 5 de 2015

Aceptado: Agosto 24 de 2015

Cómo citar:

Alfaro Rodríguez, R.; Leiva, F.R.; Gómez, M.I. 2015. Zonas homogéneas para manejo específico por sitio en maíz usando un sensor de inducción electromagnética en la sabana de Bogotá. Rev. U.D.C.A Act. \& Div. Cient. 18(2): 373-383. 